8 - ORIGINAL ARTICLE

EXPERIMENTAL ONCOLOGY

\title{
Effect of probiotics on the development of dimethylhydrazine-induced preneoplastic lesions in the mice colon ${ }^{1}$
}

\author{
Juliana Costa LiboredoI, Lucilene Rezende Anastácio"I, Maria do Carmo Gouveia Pelúzio ${ }^{\mathrm{III}}$, Flávia Xavier Valente ${ }^{\mathrm{IV}}$, Luisa \\ Costa Penna Penidov, Jacques Robert Nicolivi, Maria Isabel Toulson Davisson Correia ${ }^{\mathrm{VII}}$ \\ ${ }^{\mathrm{I}}$ Fellow PhD degree, Postgraduate Program in Food Science, Faculty of Pharmacy, UFMG, Belo Horizonte-MG, Brazil. Main author. Design and \\ conception of the study, acquisition, analysis and interpretation of data. \\ IIFellow PhD degree, Postgraduate Program in Adults Health, Medical School, UFMG, Belo Horizonte-MG, Brazil. Acquisition of data. \\ IIIPhD, Associate Professor, Department of Nutrition and Health, Viçosa Federal University, Viçosa-MG, Brazil. Supervised laboratory phases of the \\ study, acquisition of data, critical revision.

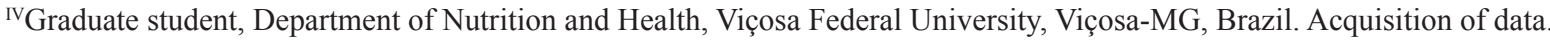 \\ ${ }^{v}$ Fellow Master degree, Department of Nutrition and Health, Viçosa Federal University, Viçosa-MG, Brazil. Acquisition of data. \\ ${ }^{v 1} \mathrm{PhD}$, Full Professor, Department of Microbiology, UFMG, Belo Horizonte-MG, Brazil. Supervised laboratory phases of the study, conception and \\ design of the study, critical revision. \\ VIIPhD, Full Professor, Department of Surgery, UFMG, Belo Horizonte-MG, Brazil. Conception and design of the study, responsible for English \\ language, critical revision.
}

\begin{abstract}
PURPOSE: To determine the effect of probiotics on the development of chemically induced (1, 2-dimethylhydrazine) colonic preneoplastic lesions, in mice.

METHODS: The animals were divided into five groups. The control group was injected with carcinogen alone and the other groups also received probiotics (1- Lactobacillus delbrueckii UFV-H2b20; 2- Bifidobacterium animalis var. lactis Bb12; 3- L. delbrueckii UFV$\mathrm{H} 2 \mathrm{~b} 20$ plus B. animalis var. lactis Bb12; and 4-Saccharomyces boulardii) administered orally in drinking water throughout fourteen weeks.

RESULTS: Consumption of lactobacilli and bifidobacteria alone resulted in a significant reduction of the total number of aberrant crypt foci $(55.7 \%$ and $45.1 \%$, respectively). Significant reduction in the number of these small foci ( $\leq 3$ aberrant crypts) was only observed in the group treated with lactobacilli (52.2\%) in comparison to control group. The number of larger foci ( $>3$ aberrant crypts) crypts had no significant reduction.

CONCLUSION: L. delbrueckii UFV-H2b20 and B. animalis var. lactis Bb12 administered alone protect colonic preneoplastic lesions in mice, while the combined treatment of these bacteria and the administration of S.boulardii were not effective in reducing such colonic lesions.
\end{abstract}

Key words: Colon. Precancerous Conditions. 1,2-Dimethylhydrazine. Probiotics. Mice. 


\section{Introduction}

The incidence of cancer is increasing worldwide, and this trend is expected to continue. New cases of cancer are estimated to rise from 11.3 million in 2007 to 15.5 million in 2030 . The number of global cancer deaths is projected to increase $45 \%$ from 2007 to 2030 (from 7.9 million to 11.5 million deaths), influenced, in part, by an increase in the global elderly population. One of the most frequent types of cancer is colorectal cancer, which has contributed significantly to increased mortality. Thus, the existence of evidence-based strategies for early detection, treatment, and especially for prevention of the disease is extremely important as approximately $40 \%$ of all cancer deaths can be prevented ${ }^{1}$. Hence, it is extremely important to implement strategies to prevent new cases of cancer.

It has been observed that the general population of intestinal bacteria is associated with initiation of colon cancer ${ }^{2}$. This has led to interest in factors that can modulate the gut microbiota and, consequently, prevent the disease. Of special interest is the beneficial effect of certain probiotics, defined as live microorganisms that, when administered in adequate amounts confer health benefit to the host ${ }^{3}$. Some studies indicate that the ingestion of certain microorganisms reduces both the risk of developing certain types of cancer and tumor growth ${ }^{4-8}$. Attention has been focused on lactobacilli and bifidobacteria. However, it is still necessary to demonstrate the effectiveness of these and other species to suppress the development of preneoplastic lesions and to determine the ideal formulation of probiotics.

The aim of the present study was to determine the effect of Lactobacillus delbrueckii UFV-H2b20, Bifidobacterium animalis var. lactis Bb12 and Saccharomyces boulardii on the prevention of pre-neoplasic lesions in mouse colon. In addition, we aimed to determine whether the combined administration of $L$. delbrueckii UFV-H2b20 and B. animalis var. lactis Bb12 would display a potential additive effect.

\section{Methods}

Eight week old male Swiss mice were distributed into five groups ( $\mathrm{n}=10 /$ group) as follows: Control group received injections of the carcinogen dimethylhydrazine; Lac group received injections of carcinogen and Lactobacillus delbrueckii UFV-H2b20; Bif group received injections of carcinogen and Bifidobacterium animalis var. lactis $\mathrm{Bb} 12$; Lac/Bif group received the carcionogen and Lactobacillus delbrueckii UFV-H2b20 plus Bifidobacterium animalis var. lactis $\mathrm{Bb} 12$; and $\mathrm{Sb}$ group received the carcinogen and Saccharomyces boulardii.

One week after the beginning of probiotic treatment, the mice were injected sub-cutaneously with the carcinogen 1,2-dimethylhydrazine (Sigma, Saint Louis, USA) (25 mg/kg, 37 mg/100 mL EDTA, s.c.) once a week, throughout six weeks.

Probiotic supplementation was administered continually throughout the entire experiment, except on the first day that DMH was administered. This is because in our pilot study, mice treated with lactobacilli, bifidobacteria, and the mixture of lactobacilli and bifidobacteria presented with $40 \%, 30 \%$, and $60 \%$ mortality, respectively, between $48 \mathrm{~h}$ and $72 \mathrm{~h}$ after the first injection of DMH. And this was related to increased bacterial translocation to mesenteric lymph nodes, spleen, and liver observed in mice injected with the carcinogen ${ }^{9}$.

On the fourteenth week of the experimental period, the mice were euthanized by cervical dislocation, under sedation, and the entire colon was collected for aberrant crypt analysis. The experimental design for this study is show in Figure 1A.

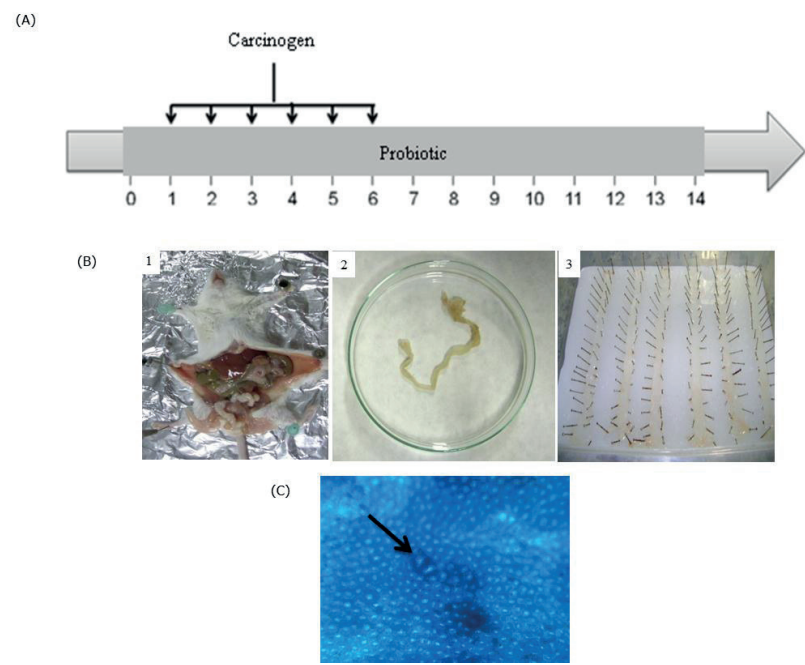

FIGURE 1 - (A) Experimental design. (B) Colon preparation for analysis of aberrant crypts: 1- removing of colon; 2- washing with saline; 3opening on paraffin plates with the mucosa facing up and fixing for 24 hours in $10 \%$ formaldehyde $(\mathbf{C})$ A dimethylhydrazine-induced aberrant crypt foci presenting two aberrant crypts methylene blue-stained (40x magnification).

The probiotics were administered orally in the water given every day at $3 \times 10^{8}$ c.f.u $\mathrm{mL}^{-1}$, except for Lac/Bif group that received $3 \times 10^{8}$ c.f.u $\mathrm{mL}^{-1}$ of each bacteria. All groups where allowed to consume water (with probiotics for the Lac, Bif, Lac/ Bif and $\mathrm{Sb}$ groups and without probiotics for the Cont group), as explained below and commercial chow (Labina, Brazil) ad libitum. The mice were housed in plastic cages with wood chips for bedding in a room with a $12 \mathrm{~h}$ light/dark cycle. The study was approved by the Ethics Committee for Animal Experimentation 
of the Universidade Federal de Minas Gerais (protocol number 189/07) and the institutional guidelines for the care and use of laboratory animals was followed.

\section{Microorganisms and preparation of water}

Lactobacillus delbrueckii UFV-H2b20 was isolated from the feces of a newborn at the Viçosa Federal University (ViçosaMG, Brazil); Bifidobacterium animalis var. lactis Bb12 was provided by the Christian Hansen Lab (Horsholm, Denmark); and Saccharomyces boulardii was provided by Merck S.A. (Rio de Janeiro, Brazil).

For the preparation of water, L. delbrueckii UFV-H2b20 and $B$. animalis var. lactis $\mathrm{Bb} 12$ were cultured for 24 hours at $37^{\circ} \mathrm{C}$ in MRS broth (Difco, Sparks, USA); S. boulardii were cultured in YPG broth (1\% yeast extract, $2 \%$ peptone, 3\% glycerol) for 48 hours at $37^{\circ} \mathrm{C}$. Bifidobacteria were incubated in an anaerobic chamber (Forma Scientific Company, Marietta, USA; containing an atmosphere of $85 \% \mathrm{~N}_{2}, 10 \% \mathrm{H}_{2}$ and $5 \% \mathrm{CO}_{2}$ ). After cultivation, the culture was centrifuged at 5,000 rpm for 15 minutes, and the supernatants were discarded. The cells were washed with $5 \mathrm{~mL}$ of water and centrifuged under the same conditions; then, the supernatants were discarded. The cells were resuspended in $100 \mathrm{~mL}$ of drinking water placed in each box with 10 animals. Fresh cultures were prepared daily and the water changed every $24 \mathrm{~h}$ when found to be about $10^{8}$ c.f.u $\mathrm{mL}^{-1}$.

\section{Aberrant Crypt Foci analysis}

At the end of the 14 week experiment, after euthanasia, the colons were immediately removed from the cecum down to the anus, rinsed in saline to remove the faeces, slit longitudinally and opened on paraffin plates with the mucosa facing up and fixed for 24 hours in $10 \%$ formaldehyde. Next, the sample was measured and divided in three equal fragments referred to as proximal, medial and distal in relation to the cecum, stained with $1 \%$ methylene blue for 2 minutes and rinsed with a phosphate buffer (PBS), in order to identify the foci of aberrant crypts. The foci throughout the entire mucosal surface of the large bowel were blindly counted by two independent and trained observers using a light microscope at $40 \mathrm{x}$ magnification according to the technique used by $\operatorname{Bird}^{10}$ (Figure 1B). Because there was no significant difference between the evaluations of the two observers ( $p>0.05)$ (data not shown), the average of two counts was used for the analysis. The total number of aberrant crypt foci (ACF), its distribution along the colon and the multiplicity of the crypts were analyzed. The distribution among the proximal, middle and distal colon was evaluated, and multiplicity was determined by counting the foci containing three or fewer aberrant crypts and foci with more than three aberrant.

\section{Statistical analysis}

Data were analyzed using the SigmaPlot (Version 11.0). Student's t-test was utilized to compare the counts of two observers and for further analysis of aberrant crypts between groups treated with probiotics and the Cont group. Analysis of ACF in the proximal, middle and distal regions of mouse colon was carried out using ANOVA followed by Holm-Sidak test. The differences were considered significant at $\mathrm{p}<0.05$.

\section{Results}

There were no significant differences in the initial and after 14 weeks body weight and food intake among the experimental groups (data not shown).

All mice developed ACF in the colon (Figure 1C). Data on the total number of ACF, multiplicity of aberrant crypts and the distribution of ACF in the colon after 14 weeks of treatment in the different groups are summarized in Table 1.

TABLE 1 - Effects of on the formation of aberrant crypt foci (ACF) induced by DMH in the mice colon.

\begin{tabular}{|c|c|c|c|c|c|c|}
\hline & \multirow{2}{*}{ Total no. of ACF } & \multicolumn{2}{|c|}{ No. of ACF with } & \multicolumn{3}{|c|}{ Distribution of $\mathrm{ACF}$ in the colon } \\
\hline & & $\leq 3$ crypts & $>3$ crypts & proximal & middle & distal \\
\hline Cont & $62.0 \pm 7.9$ & $58.6 \pm 4.0$ & $2.2 \pm 1.6$ & $10.5 \pm 1.1^{\#}$ & $21.7 \pm 3.7$ & $24.9 \pm 3.4$ \\
\hline Bif & $34.0 \pm 7.8^{*}$ & $56.7 \pm 8.9$ & $0.6 \pm 0.5$ & $16.4 \pm 0.8$ & $18.1 \pm 3.5$ & $16.5 \pm 5.4$ \\
\hline Lac/Bif & $81.0 \pm 24.9$ & $79.6 \pm 27.4$ & $2.1 \pm 1.0$ & $19.2 \pm 8.3$ & $22.1 \pm 0.7$ & $30.7 \pm 1.4^{\#}$ \\
\hline Sb & $58.0 \pm 4.5$ & $58.1 \pm 7.9$ & $1.8 \pm 0.7$ & $26.2 \pm 3.6^{\#}$ & $15.0 \pm 1.2$ & $15.1 \pm 1.3$ \\
\hline
\end{tabular}

Data are presented as the mean $\pm \mathrm{SD} .^{*}, \mathrm{p}<0.05$, the group was significantly different from Cont group. \#, $\mathrm{p}<0.05$, total number of ACF was significantly different of the other parts of the colon, in each group. 
The mean number of ACF per colon in the DMH alone group (Cont) was $62.0 \pm 7.9$ whereas mice treated with Lactobacillus and Bifidobacteria showed a significantly lower number of ACF. A $55.7 \%$ and $45.1 \%$ reduction of ACF was found in the Lac and Bif groups, respectively. However, we did not find a synergistic effect of Lactobacillus plus Bifidobacteria in the inhibition of DMH-induced ACF formation. Moreover, the number in the $\mathrm{Sb}$ group was reduced only by $6.12 \%$ compared to the Cont group. The reduced ACF occurred from the middle to distal part of the colon of animals treated with probiotics (except in the Lac/Bif group) compared to DMH alone group.

Crypt multiplicity was suppressed in all groups treated with probiotics. The majority of the ACF comprised one to three aberrant crypts. Significant reduction in the number of these small ACF was only observed in the Lac $(52.2 \% ; 28.0 \pm 6.04)$ and was slightly reduced in the Bif $(3.3 \% ; 56.7 \pm 8.9)$ and SB groups $(0.8 \% ; 58.1 \pm 7.8)$ in comparison to mice treated with the carcinogen alone $(58.6 \pm 4.0)$. Curiously, an increase in ACF was observed in the Lac/Bif group (35.8\%; 79.6 \pm 27.4$)$.

The most potent protective effect against large ACF was observed in the Bif group, in which the number of foci with more than three aberrant crypts was decreased by $74 \%(0.6 \pm 0.5)$, followed by reductions of $21.5 \%(1.7 \pm 0.8), 21.1 \%(1.8 \pm 0.7)$ and $5.8 \%(2.1 \pm 1)$ in the Lac, $\mathrm{Sb}$ and Lac/Bif groups, respectively (Figure 2).

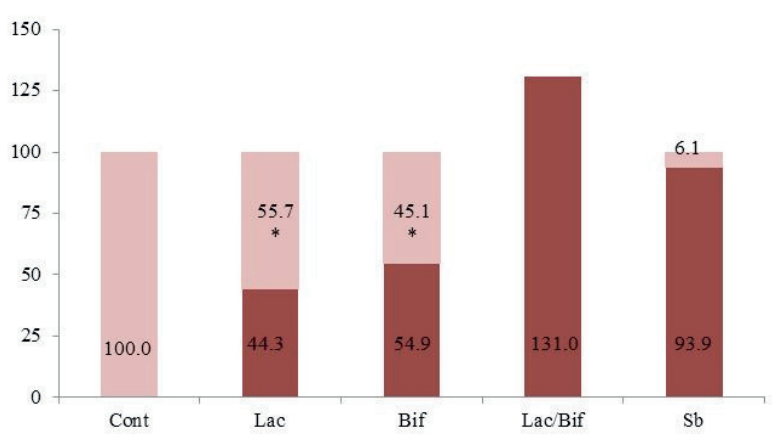

(B)

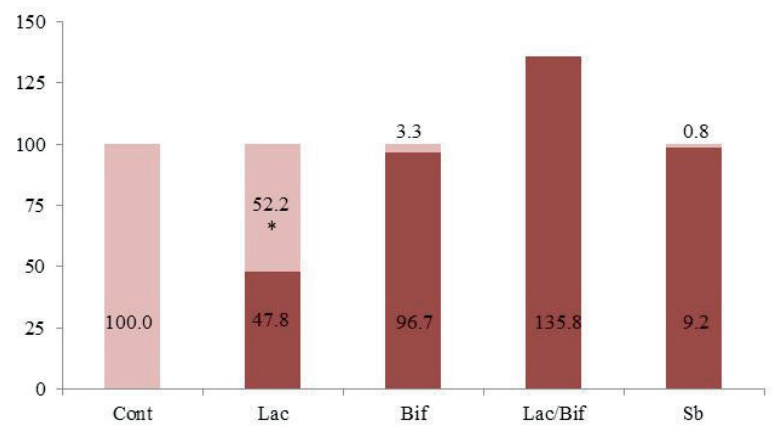

(C)

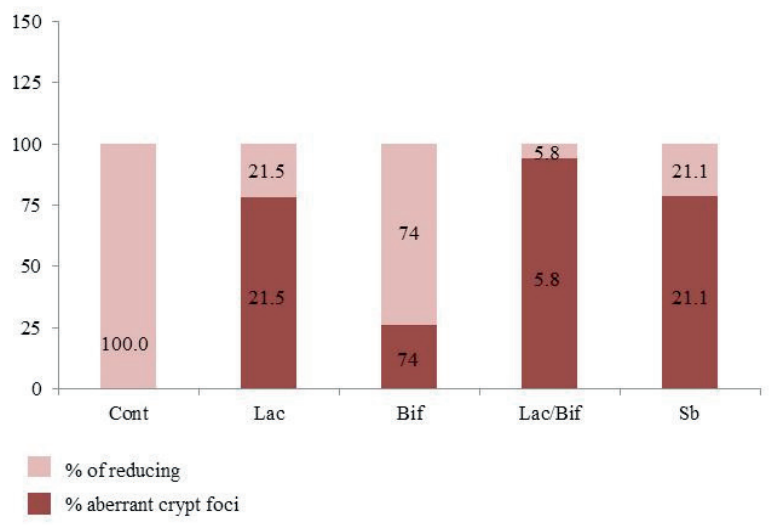

FIGURE 2-Percentage of aberrant crypt foci induced by dimethylhydrazine in the mice colon and percentual of reduction of aberrant crypt foci in groups treated with Lactobacillus delbrueckii UFV-H2B20 (Lacto), Bifidobacterium animalis var. lactis Bb12 (Bifido), L. delbrueckii UFV$\mathrm{H} 2 \mathrm{~B} 20$ plus B. animalis var. lactis Bb12 (Lacto/Bifido) and Saccharomyces boulardii $(\mathrm{Sb})$ in relation to control group. FIGURE 2 - Percentage of aberrant crypt foci induced by dimethylhydrazine in the mice colon and percentual of reduction of aberrant crypt foci in groups treated with Lactobacillus delbrueckii UFV-H2B20 (Lacto), Bifidobacterium animalis var. lactis Bb12 (Bifido), L. delbrueckii UFV-H2B20 plus B. animalis var. lactis $\mathrm{Bb} 12$ (Lacto/Bifido) and Saccharomyces boulardii (Sb) in relation to control group. Values were obtained in relation to average of aberrant crypt foci found in the Cont group, considered as $100 \%{ }^{*}, p<0.05$, the group was significantly different from Cont group. (A) Total of aberrant crypt foci; (B) Foci containing three or fewer aberrant crypts; (C) Foci with more than three aberrant crypts.

\section{Discussion}

In the present study, we demonstrate that consumption of Lactobacillus delbrueckii UFV-H2B20 and Bifidobacterium animalis var. lactis $\mathrm{Bb} 12$ resulted in significant inhibition of DMH- 
induced ACF formation in mice, indicating a chemopreventive efficacy of these probiotics against colonic tumorigenesis, a shortterm effect and without any noticeable adverse effects. Although the mechanism of inhibition of colon cancer by probiotics is not clear, we believe that this effect may proceed through diverse mechanisms, including alteration of intestinal microbiota; estimulation of immune response and inactivation of carcinogenic compounds.

Other studies in animal models of colon cancer have also shown that the consumption of some probiotics could inhibit preneoplastic lesions and tumor development. An important reduction was observed in azoxymethane-induced ACF and colon crypt multiplicity after animals were fed $B$. longum ${ }^{11}$. Bolognani et $a l^{7}$ observed a significant decrease in azoxymethaneinduced colonic ACF in rats given L. acidophilus. A symbiotic combination was also demonstrated to have a beneficial effect, observed by the reduction in ACF with the use of inulin and $B$. longum $^{4}$ or of oligofructose and bifidobacteria ${ }^{5}$. In another study, the combined administration of B. lactis and L. rhamnosus with inulin and oligofructose in rats was able to reduce the occurrence of malignant tumors induced by the carcinogen azoxymethane ${ }^{8}$.

Previous studies also showed inhibition of preneoplastic lesions and tumors as a result of Lactobacillus plus Bifidobacterium supplementation $\left(10^{9} \mathrm{CFU} / \mathrm{mL}\right)^{12-14}$. In the current study, $L$. delbrueckii UFV-H2B20 and $B$. animalis var. lactis $\mathrm{Bb} 12$ supplementation separately reduced the total number of ACF. We also used a mixture containing $10^{8} \mathrm{CFU} / \mathrm{mL}$ of each bacteria (L. delbrueckii UFV-H2B20 plus B. animalis var. lactis Bb12) to verify whether feeding mice a large amount of a combination of bacterial species would exert better effects than the use of a single species or the use of a small amount. However, we were not able to demonstrate the positive effects of such a combination. It was not possible to determine the reason why the combination of probiotics showed no positive result, but it's our impression that this may be due to the used dose that may have caused a derangement in the intestinal microbiota or in the immune system.

Aberrant crypt foci, that are enlarged and elevated relative to normal crypts, have also been detected in both rodents and humans and can be recognized as early neoplastic lesions. Furthermore, crypt multiplicity (number of crypts per focus) has been considered to be one of the consistent predictors of tumor incidence. It has been suggested that large $\mathrm{ACF}$ are more predictive of tumor formation than small $\mathrm{ACF}^{15}$. In the present study, we observed that small ACF were predominant in all groups. The formation of small ACF was mostly inhibited in the Lac group, while the most potent inhibition of large ACF was observed in the
Bif group, when both were compared to the Cont group. However, the reduction seen in the Bif group was not significant, probably due to the high standard deviation.

In addition to crypt multiplicity, the location of ACF is also an interesting feature. In this study, the majority of ACF was present in the medial and distal colon in the Cont/DMH group. According to the literature, in both human and animal colons, ACF are located in distal segments more often than in proximal regions, a characteristic also observed in colorectal cancers ${ }^{16}$. Interestingly, the reduction of ACF occurred in middle and distal regions in all groups treated with probiotics, except in Lac/Bif group that showed no significant positive effect.

To our knowledge, only one study has evaluated the effect of $S$. boulardii in cancer and analyzed its effect on tumor development. In this work, Chen et al ${ }^{17}$ observed the prevention of cancer cell formation through the reduction of epidermal growth factor-mediated cell proliferation and increased apoptosis, as well as by a $50 \%$ decrease in tumor number and volume in the distal small intestine of C57BL/6J Min/+ $\left(A p c^{M i n}\right)$ mice treated with $S$. boulardii. These results pointed to the potential role of the yeast in the prevention or treatment of intestinal adenomatous polyps and/ or adenocarcinoma. S. boulardii is a yeast that has been widely used as a probiotic agent in human medicine, and its efficacy in the prevention and treatment of various intestinal disorders has been demonstrated ${ }^{18,19}$. However, in the present study, S. boulardii did not exert a marked effect on the formation of preneoplastic lesions. The best result was observed in the reduced number of ACF with more than three aberrant crypts $(21.07 \%)$ in the group receiving $S$. boulardii as compared to the Cont/DMH group, but the difference was not significant.

In summary, our study suggests that only certain probiotic bacteria (L. delbrueckii UFV-H2b20 and B. animalis var. lactis Bb12) are capable of diminishing preneoplastic lesions development carcinogen-induced, but this beneficial effect seems to be related, under the experimental conditions, to the type of microorganism used. Our results confirm what has been pointed out by Azcárate-Peril et $a .^{20}$ in their review on the beneficial effects of probiotics in prevention of colorectal cancer, that the role of probiotic is species and strain dependent.

More probiotic studies are needed to examine possible mechanisms for their potential benefit.

\section{Conclusion}

Among the three tested probiotics, only L. delbrueckii UFV-H2b20 and B. animalis var. lactis Bb12 administered alone 
protected against colon carcinogenesis.

\section{References}

1. World Health Organization. Cancer. Available from: $<$ http://www. who.int/cancer/en/>. Access in November 18, 2008.

2. Reddy BS, Narisawa T, Wright P, Vukusich D, Weisburger JH, Wynder EL. Colon carcinogenesis with azoxymethane and dimethylhydrazine in germ-free rats. Cancer Res. 1975;35(2):28790.

3. FAO/WHO Expert Consultation: "Guidelines for the Evaluation of Probiotics in Food" London, Ontario (Canada), 2002.

4. Rowland IR, Rumney CJ, Coutts JT, Lievense LC. Effect of Bifidobacterium longum and inulin on gut bacterial metabolism and carcinogen-induced aberrant crypt foci in rats. Carcinogenesis. 1998;19:281-5.

5. Gallaher DD, Kill J. The effect of synbiotics on colon carcinogenesis in rats. J Nutr. 1999;129(Suppl. 7):1483-7.

6. Le Leu RK, Hu Y, Brown IL, Woodman RJ, Young GP. Synbiotic intervention of Bifidobacterium lactis and resistant starch protects against colorectal cancer development in rats. Carcinogenesis. 2010;31(2):246-51.

7. Bolognani F, Rumney CJ, Pool-Zobel BL, Rowland IR. Effect of lactobacilli, bifidobacteria and inulin on the formation of aberrant crypt foci in rats. Eur J Nutr. 2011;40(6):293-300.

8. Femia AP, Luceri C, Dolara P, Giannini A, Biggeri A, Salvadori M, Clune Y, Collins KJ, Paglierani M, Caderni G. Antitumorigenic activity of the prebiotic inulin enriched with oligofructose in combination with the probiotics Lactobacillus rhamnosus and Bifidobacterium lactis on azoxymethane induced colon carcinogenesis in rats. Carcinogenesis. 2002;23(11):1953-60.

9. Liboredo JC, Anastácio LR, Mattos LV, Nicoli JR, Correia MITD. Impact of probiotic supplementation on mortality of induced 1,2-dimethylhydrazine carcinogenesis in a mouse model. Nutrition. 2010;26:779-83.

10. Bird RP. Observation and quantification of aberrant crypts in the murine colon treated with a colon carcinogen: preliminary findings. Cancer Lett. 1987;37(2):147-51.

11. Kulkarni N, Reddy BS. Inhibitory effect of Bifidobacterium longum cultures on the azoxymethane-induced aberrant crypt foci formation and fecal bacterial b-glucuronidase. Proc Soc Exp Biol Med. 1994;207(3):278-83.

12. Mcintosh GH, Royle PJ, Playne MJ. A probiotic strain of Lactobacillus acidophilus reduces DMH-induced large intestinal tumors in male Sprague Dawley rats. Nutr Cancer. 1999;35(2):1539.

13. Foo N-P, Yang HO, Chiu H-H, Chan H-Y, Liao C-C, Yu C-K, Wang Y-J. Probiotics prevent the development of 1,2-dimethylhydrazine $(\mathrm{DMH})$-induced colonic tumorigenesis through suppressed colonic mucosa cellular proliferation and increased stimulation of macrophages. J Agric Food Chem. 2011;59:13337-45.

14. Pretlow TP, Barrow BJ, Ashton WS, O'riordan MA, Pretlow TG, Jurcisek JA, Stellato TA. Aberrant crypts: putative preneoplastic foci in human colonic mucosa. Cancer Res. 1991;51(5):1564-7.

15. Rodrigues MAM, Silva LAG, Salvadori DMF, Camargo JLV, Montenegro MR. Aberrant crypt foci and colon cancer: comparison between a short- and medium-term bioassay for colon carcinogenesis using dimethylhydrazine in Wistar rats. Braz J Med Biol Res. 2002;35(3):351-5.

16. Chen X, Fruehauf J, Goldsmith JD, Xu H, Katchar KK, Koon H-W, Zhao D, Kokkotou EG, Pothoulakis C, Kelly CP. Saccharomyces boulardii inhibits EGF receptor signaling and intestinal tumor growth in Apc(min) mice. Gastroenterology. 2009;137(3):914-23.

17. Surawicz CM. Probiotics, antibiotic-associated diarrhea and Clostridium difficile diarrhea in humans. Best Pract Res Clin Gastroenterol. 2003;17(5):775-83.

18. Guslandi M, Giollo P, Testoni PA. A pilot trial of Saccharomyces boulardii in ulcerative colitis. Eur J Gastroenterol Hepatol. 2003;15(6):697-8.

19. Azcárate-Peril MA, Sikes M, Bruno-Bárcena JM. The intestinal microbiota, gastrointestinal environment and colorectal cancer: a putative role for probiotics in prevention of colorectal cancer? Am J Physiol Gastrointest Liver Physiol. 2011;301:G401-G24.

\section{Correspondence:}

Maria Isabel Toulson Davisson Correia

Departamento de Cirurgia, Faculdade de Medicina-UFMG

Avenida Carandaí, 246/902

310130-060 Belo Horizonte - MG Brasil

Tel.: (55 31)9168-8239

Fax: (55 31)3241-1367

isabel_correia@uol.com.br

Received: January 15, 2013

Review: March 14, 2013

Accepted: April 16, 2013

Conflict of interest: none

Financial sources: Coordination of Improvement for Higher Academic Staff (CAPES) and National Council of Technological and Scientific Development $(\mathrm{CNPq})$

${ }^{1}$ Research performed at Laboratory of Ecology and Physiology of Microorganisms, Department of Microbiology, Institute of Biological Sciences, Federal University of Minas Gerais (UFMG), Brazil. Part of Master degree thesis, Postgraduate Program in Food Science, Faculty of Pharmacy, UFMG. Tutor: Maria Isabel Toulson Davisson Correia. 